\title{
A rare presentation of orbital complication of invasive fungal sinusitis in an immunocompetent young boy-a rare case
}

S. Gaur ${ }^{1}$, A. Lavania, ${ }^{2}$ R. Saxena ${ }^{3}$

${ }^{1}$ Senior Resident, ${ }^{2}$ Associate Professor, ${ }^{3}$ Professor

Department of E.N.T and Head \& Neck Surgery, School of Medical Science \& Research, Greater Noida, Uttarpradesh, India

\begin{abstract}
We present a case of allergic fungal sinusitis (AFS) in a 24 -year old man with history of left sided nasal obstruction and discharge since few years. Since few months he developed epiphora in the left eye associated with discomfort on eye movements. Patient was examined and CT with contrast was done. CT contrast showed an enhancing lesion in Left maxillary and ethmoid sinuses and erosion of the inferior bony wall of the orbit and medial wall of maxillary sinus.
\end{abstract}

Though most patients of fungal sinusitis are immunocompromised but this patient was young male immunocompetent and made an unusual presentation with visual epiphora and painful eye movements. CT showed bony erosion of the Left inferior Bony wall of the Orbit and medial wall of Maxillary Sinus.

After through examination and specific investigations, the patient was posted for surgery. We planed for Cald well-Luc's Surgery and Endoscopic excision of the mass .Histological examination was reported as non malignant and microscopy showed Fungal Hyphae.

After the surgery patient was discharged satisfactorily within couple of days and followed up regularly.

Key words: Aspergillosis, fungal sinusitis, immunocompetent.

\section{Introduction}

Four distinct histologic categories of fungal sinusitis (FS) have been recognized: allergic, noninvasive fungal colonization (mycetoma or "fungal ball"); chronic invasive; and acute fulminant. Histological features, which were highly sensitive and specific for the diagnosis of allergic FS, included: eosinophilic mucin with Charcot-Leyden crystals and collections of

Correspondence:A. Lavania

E-mail: ajayisdiamond@rediffmail.com degenerating eosinophils ${ }^{1}$. Allergic mucin is very characteristic but not pathognomonic for allergic fungal sinusitis.

In chronic noninvasive fungal sinusitis, the fungi themselves consist of hyphae without spores and show septation with branching at $45^{\circ}$ angles. These fungal balls are easily identifiable by Hematoxylin and Eosin staining $(\mathrm{H} \& \mathrm{E})$ and do not invade mucosal tissue in 
S. Gaur, A rare presentation of orbital complication of invasive fungal

any case. In contrast to the case of allergic fungal sinusitis, mucin is scant or absent in these cases.

In chronic invasive fungal sinusitis a nonspecific, non necrotizing granulomas, which interestingly contains eosinophils is commonly seen, no fungal hyphae are found within these mucosal granulomas.

In acute invasive fungal sinusitis vascular invasion by numerous fungal hyphae are present.

Thus special stains for fungi should always be performed when allergic mucin is seen, which reveals fungal hyphae in $\mathrm{d}^{\prime} 85 \%$ of cases. ${ }^{2-3}$

We present here a case of allergic fungal sinusitis (AFB), who underwent maxillary antrostomy due to the mass in paranasal sinuses.

\section{Case report}

A 24 -year old man presented to E.N.T Out patient Department of School of Medical Science and Research, Greater Noida with the history of left sided nasal obstruction and discharge since few years. And he developed epiphora in the left eye associated with discomfort on eye movements since few months. On Examination left eye movements were restricted while vision was Normal in both the eyes. Anterior \& Posterior Rhinoscopy examination revealed pale nasal mucosa and small necrotic mass in left nasal cavity. CT with contrast was done .CT contrast showed an enhancing lesion in Left maxillary and ethmoid sinuses and erosion of the inferior bony wall of the orbit and medial wall of maxillary sinus (Fig 1).

A patient made an unusual presentation with visual epiphora and painful eye movements. Erosive bony landmarks of sinuses in the CT scan raised the serious doubt of Invasive Fungal sinusitis (Fig 1).
After thorough examination and specific investigations, the patient was posted for surgery. We planed for Cald well-Luc's Surgery and Endoscopic excision (Combined approach) of the mass.

The thick blackish gelatinous mass was removed in total from the maxillary sinuses. And Endoscopically the polypoidal mass arising from the lateral wall of the nose was removed. Medial wall and roof of the maxillary sinus and was grossly eroded.

Histological examination was reported as non malignant and microscopy showed Fungal hyphae infiltrating the bony lacunae.(Fig 2) After the surgery patient was discharged satisfactorily within couple of days and followed up regularly.

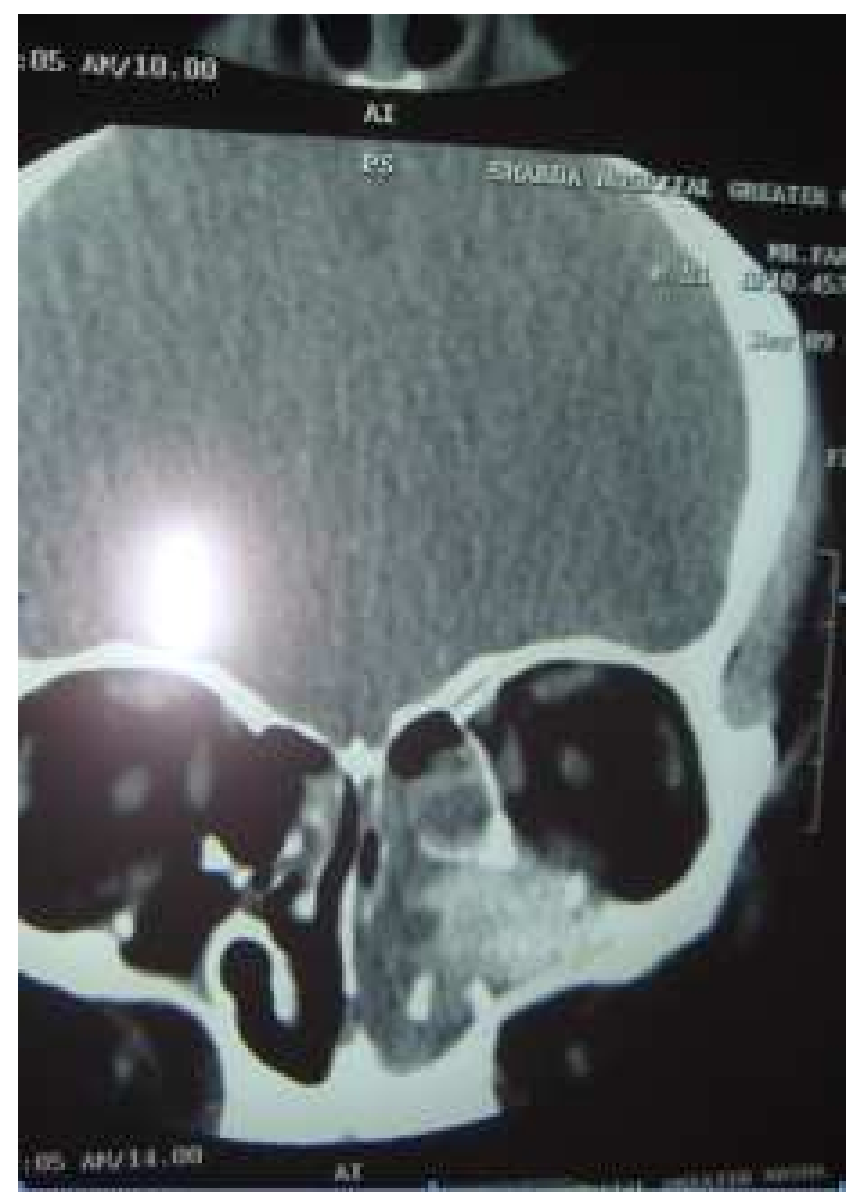

Fig. 1: CT Scan of the patient showing hypodense, expansile mass invading the maxillary, ethmoid sinus and eroding the roof and medial wall of left orbit 


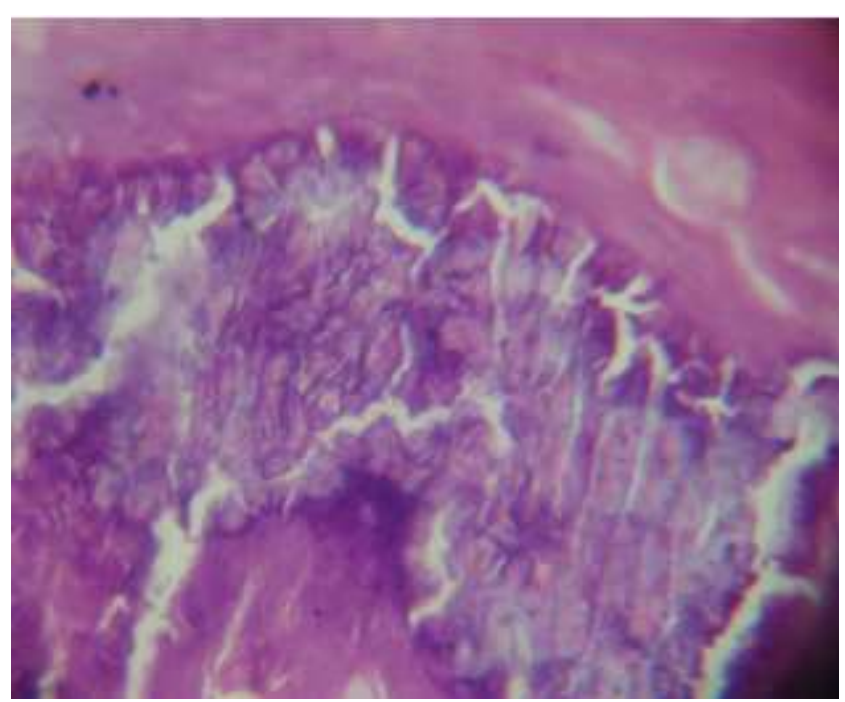

Fig. 2: Microphotograph from curetted material of Maxillary sinus shows fungal ball of Aspergillus infiltrating inside the bony Lacunae (H\&E Stain X400)

\section{Discussion}

Four distinct histologic categories of fungal sinusitis have been recognized: allergic, noninvasive fungal colonization (mycetoma or "fungal ball"); chronic invasive; and acute fulminant.

In chronic noninvasive fungal sinusitis, fungal balls are easily identifiable by $\mathrm{H} \& \mathrm{E}$ staining and do not invade mucosal tissue in any case. In chronic invasive fungal sinusitis a nonspecific, non necrotizing granulomas are found and no fungal hyphae are found within these mucosal granulomas ${ }^{4}$. In acute invasive fungal sinusitis vascular invasion by numerous fungal hyphae are present.

Invasive fungal disease with rare exception, occurs in severely immunodeficient patients ${ }^{5}$. Diabetic patients experiencing ketoacidosis are at risk for developing rhinocereberal infection from fungi. Profoundly neutropoenic patients after transplant or chemotherapy for malignancy are also at risk for invasive fungal sinusitis, usually with aspergilus species. ${ }^{6}$ The diagnosis of invasive fungal sinusitis is dependent upon high index of suspicion in a patient with signs such as fever, nasal congestion and discharge, paresthetic facial pains, and erythema around the nostrils or lower eyelids. Nasal swabs demonstrating fungal Hyphae are highly suggestive, but diagnosis ultimately depends on biopsy 5 .

The treatment of invasive fungal sinusitis ultimately depends on reversing the diabetic Ketoacidosis or improving the immune status or transplant or cancer patients. ${ }^{7}$ The later is especially difficult, accounting for the high mortality rate in the patientgroup. Immunomodulation and hyperbaric oxygen have also been tried with mixed results. Anti-leukotrienes are also being for used. ${ }^{8}$ Surgery is important both for initial diagnosis and for excision of necrotic tissue, allowing for better treatment efficacy of Amphotericin B. ${ }^{9}$

Therefore, some clinicians are now advocating more conservative endoscopic serial debridement of necrotic tissue more often than radical maxillectomy with orbital exentration.

\section{Conclusion}

Chronic Invasive fungal sinusitis may effect immunocompetent healthy patient with orbital involvement. Surgical debridement and empiric systemic antifungal therapy, followed by serial endoscopic evaluations, are the mainstays of treatment. Early diagnosis can prevent the extensive surgical intervention. we present here such a case who was managed satisfactorily and followed up at regular interval. 
S. Gaur, A rare presentation of orbital complication of invasive fungal

\section{References}

1. J.F. Lara, J.D. Gomez. Allergic mucin with and without fungus: A comparative clinicopathologic analysis. Arch Pathol Lab Med 2001;125(11):1442-7.

2. L. Granville, M. Chirala, P. Cernoch et al. Fungal sinusitis: histologic spectrum and correlation with culture. Hum Pathol 2004;35(4):474-81.

3. J.U. Ponikau, D.A. Sherris, E.B. Kern. et al .The Diagnosis and incidence of allergic fungal sinusitis. Mayo Clin Proc 1999;74(9):877-84.

4. M. Brandwein, Histopathology of sinonasal fungal disease. Otolaryngol Clin North Am 1993; 26(6):949-81.

5. P.P. Bhadani, U.K. Bhadani, N. Thapliyal et al. rare presentation of invasive rhino-orbital mucormycosis in an immunocompetent young girl: a case report. Indian J Pathol Microbiol. 2007;50(4):785-6.

6. Mohamad Javed Ashraf,Negar Azarpira,Mehrzad Pourjafar,and Bijan Khademi Allergic Fungal Sinusitis presenting as a paranasal Sinus Tumor:Case Report. Iran.J. Allergy Asthma Immunol December 2005;4 (4):193-95.

7. M.S. Schubert, D.W. Goetz. Evaluation and Treatment of Allergic Fungal Sinusitis.II Treatment and Followup. J Allergy Clin Immunol 1998;102(3):395-402.

8. M.S. Schubert. Antileukotriene Therapy for allergic Fungal sinusitis. $J$ Allergy Clin Immunol 2001;108(3):466-7.

9. D.P. Kontoyiannis, G.P. Bodey. Invasive aspergillosis in 2002: an update. Eur J Clin Microbiol Infect Dis 2002; 21: 161-72. 